\title{
Comparison of GreenLight 180-W XPS laser vaporization versus transurethral resection of the prostate: Outcomes of a single regional center
}

\author{
Daniele Mattevi, Lorenzo Luciani, Rosa Spina, Claudio Divan, Stefania Cicuto, Tommaso Cai, \\ Valentino Vattovani, Marco Puglisi, Stefano Chiodini, Gianni Malossini \\ Department of Urology, Santa Chiara Hospital, Trento, Italy.
}

\begin{abstract}
Summary Background: To evaluate the intermediate perioperative outcomes, rate of complications and functional data after XPS 180-W Greenlight photoselective laser vaporization (PVP) compared with transurethral resection of the prostate (TURP) in a prospective non-randomized single centre study.

Methods: We analyzed a prospectively-maintained database collecting data on 100 patients undergoing surgical treatment of BPH (50 consecutive PVP and 50 consecutive TURP). All complications, recorded and graduated according to the Clavien Dindo system and the clinical, operative, perioperative variables were compared. The functional outcomes,

International Prostate Symptom Score (IPSS), max flow rate $\left(Q_{\max }\right)$ and Prostate Specific Antigen (PSA), were recorded preoperatively and at 1 year of follow up.

Results: Age, prostate volume, use of anticoagulants or antiplatelets, ASA score and operative time were comparable in the two groups. The reduction in the hemoglobin levels ( 0.46 vs $1.8 \mathrm{~g} / \mathrm{dL})$, the catheterization time (1.2 vs 3.2 days), the hospital stay (1.7 vs 3.8 days) and rate of transfused patients (0 vs 8\%), were significantly lower for PVP. Transient re-catherization (6 vs 26\%) was significantly lower for PVP.

The IPSS and $Q_{\text {max }}$ at 1 year showed no significant difference. The rate of repeat TURP/PVP was higher in the TURP group (0 vs 10\%). Reduction of PSA, that reflects the major reduction of prostate volume, was statistically greater in PVP group respect TURP group $(p=0.001)$.

Conclusions: PVP has advantages in terms of perioperative safety and major complications than TURP. Functional outcomes at 1 year of follow-up were comparable.
\end{abstract}

KEY WORDS: GreenLight; TURP; Photoselective vaporization; Laser; Benign prostatic hyperplasia.

Submitted 22 April 2020; Accepted 31 May 2020

\section{INTRODUCTION}

Benign prostatic hyperplasia (BPH) is a very common disease among elderly men. This pathology leads the patient to have symptoms of the lower urinary tract (LUTS) and lowers the quality of life (1). Medical therapy is usually the first-line treatment (2). Thus, more invasive treatment options have to be considered as the disease progresses. Transurethral resection of the prostate (TURP) is still considered the gold standard for the treatment of LUTS (3), which improves the urinary flow rate and reduces the voiding symptoms (4). However, this tech- nique has complications $(5,6)$, such as the transurethral resection syndrome secondary to irrigation fluid absorption, which presents risk factors including bleeding, operative times and large prostatic volume $(7,8)$. Laser treatment of the prostate is acquiring a role as a minimally invasive treatment of BPH. Potassium tytanyl phosphate (KTP) laser for the treatment of BPH was first used by Watson in 1995 (9). Over the years the GreenLight laser has evolved starting from a power of $80 \mathrm{~W}$, passing through a system to $120 \mathrm{~W}$ (GL-HPS) up to the $180 \mathrm{~W}$ XPS (GL-XPS) in 2011. The increase in power has made it is also necessary to introduce new laser fibers for an efficient ablation of prostate tissue (10). The light beam KTP $532 \mathrm{~nm}$ is selectively absorbed by oxyhemoglobin, facilitating coagulation and vaporization of tissues. After its introduction, the technique was confirmed as effective and safe in the short and medium term (11, 12). Furthermore, it has been shown that the procedure does not require the anticoagulant suspension because the procedure is almost bloodless, making the procedure optimal in high-risk patients (13).

In the present study, we report our experience with photoselective vaporization of the prostate (PVP) and TURP regarding complications and functional results with a follow up to 1 year.

\section{Material AND METHOdS}

We analyzed a prospectively-maintained database collecting data on 100 patients undergoing surgical treatment of BPH (50 consecutive PVP and 50 consecutive TURP) from March 2015 to March 2016 at the Department of Urology of Trento, Italy. The clinical, operative, perioperative, variables are shown in Table 1. All complications that occurred within 30 days of discharge and within 1 year have been recorded, defined and graduated according to the Clavien Dindo system (14) (Table 2). Comorbidities were evaluated according to preoperative risk assessment by the American Society of Anesthesiologist (ASA score).

The functional results were summarized in Table 3: International Prostate Symptom Score (IPSS); max flow rate $\left(\mathrm{Q}_{\max }\right)$ and Prostate Specific Antigen (PSA). This data were recorded preoperatively and at 1 year of follow up. Informed consent was obtained from all individual participants included in the study. 


\section{Surgical technique}

The TURP was performed by two urologists and the PVP was performed by two other urologists. TURP was performed with a 26-F resectoscope; at the end, a bladder catheter $22 \mathrm{Ch}$ was placed with continuous bladder washing. The PVP was performed using the GreenLight XPS 180-W laser that uses a lithium tribal crystal instead of the KTP crystal, to produce a more collimated and powerful $532 \mathrm{~nm}$ laser beam than $80 \mathrm{~W}$. This results in an intervention faster and greater ability to penetrate into prostate tissue (15). From the veru montanum to the bladder neck, the median and lateral lobes were vaporized with a final result similar to a TURP.

\section{Statistical analysis}

Distribution of continuous variables are reported as mean and standard deviation. Categorical variables are presented as numbers and percentages. The comparison between subgroups (surgical approach) was performed using Student $t$ test or Mann-Whitney U test for continuous variables. Qualitative data were compared by the $X^{2}$ test or Spearman correlation. P values were considered significant when less than or equal to 0.05. All analyses were performed using the SPSS software (IBM Corp., Armonk, NY).

\section{RESULTS}

100 consecutive patients underwent endoscopic surgical treatment of BPH from March 2015 to March 2016: 50 in the PVP and 50 in the TURP groups. Demographic, preoperative, operative and postoperative characteristics are summarized in Table 1: Age, prostate volume, use of anticoagulants or antiplatelets, and ASA score were comparable between groups. Also, the mean operative time was comparable in the two groups. Compared to preoperative values, there was a significant reduction in the hemoglobin levels at the end of TURP procedure as compared to PVP. The average duration of catheterization was $1.2 \pm 0.5$ days for PVP and $3.1 \pm 3.4$ days for TURP group ( $<<$ 0.001 ) and also the definitive catheter removal was in favor of PVP $(1.4 \pm 0.8$ vs $5.6 \pm 6.6 ; p<0.001)$. Hospital stay was also statistically shorter in the PVP as compared to TURP (2 days less). During PVP no blood transfusions were necessary whereas 4 patients (8\%) undergoing TURP were transfused due to a significant bleeding ( 2 intraoperative and 2 postoperative $)(\mathrm{p}=0.04)$.

Early ( $<30$ days) and late complications are presented in Table 2. Transient recatherization was performed for urinary retention at catheter removal in 13 (26\%) of patients undergoing TURP and in $3(6 \%)$ undergoing PVP $(p=0.01)$. Five patients $(10 \%)$ with bladder neck sclerosis or prostate tissue regrowth requiring reTURP were found in the TURP group; none were found in the PVP group $(p=0.02)$. Two cases of mild incontinence were found in TURP group. The complications were graduated according to the classification of ClavienDindo and subdivided in minor and major complications. No major complications were found in PVP group, while 7 occurred in TURP group $(\mathrm{p}=0.01)$. Overall complications were comparable in two groups.

Efficacy in terms of IPSS, $\mathrm{Q}_{\max }$ and reduction of PSA are shown in Table 3. At 1 years, the outcome for both pro-
Table 1.

Preoperative, and early postoperative outcomes in the two study groups.

\begin{tabular}{|lccc|}
\hline & TURP $(\mathbf{n}=\mathbf{5 0})$ & $\mathbf{P V P}(\mathbf{n}=\mathbf{5 0})$ & $\mathbf{p}$ value \\
\hline Age (years) & $68.8 \pm 11.1$ & $71.6 \pm 7.4$ & 0.14 \\
\hline Prostate volume (cc) & $48.1 \pm 14.5$ & $53.3 \pm 14.7$ & 0.08 \\
\hline Anticoagulans/antiplatelet $(\mathrm{n})$ & 18 & 19 & 0.83 \\
\hline ASA Score 3 ( $\mathrm{n})$ & 11 & 17 & 0.18 \\
\hline Operative time (min) & $61.6 \pm 27.9$ & $63 \pm 15.1$ & 0.8 \\
\hline Catheterization time (days) & $3.1 \pm 3.4$ & $1.2 \pm 0.5$ & 0.001 \\
\hline Definitive catheter removal (days) & $5.6 \pm 6.6$ & $1.4 \pm 0.8$ & 0.001 \\
\hline Tranfused patients (n) & 4 & 0 & 0.04 \\
\hline $\begin{array}{l}\text { Hospital stay (days) } \\
\text { Haemoglobin (8/L) } \\
\text { preoperative } \\
\text { first day }\end{array}$ & $3.8 \pm 2.6$ & $1.7 \pm 0.8$ & 0.001 \\
hemoglobine reduction & $14.4 \pm 1.3$ & $13.7 \pm 1.4$ & \\
\hline
\end{tabular}

Table 2.

Early and late postoperative complications.

\begin{tabular}{|c|c|c|c|}
\hline & TURP $(n=50)$ & $\operatorname{PVP}(n=50)$ & $\mathrm{p}$ value \\
\hline \multicolumn{4}{|c|}{ Early postoperative complications (<30 days) } \\
\hline Clot retention (n) & 13 & 3 & 0.01 \\
\hline Dysuria/urge (n) & 8 & 16 & 0.06 \\
\hline \multicolumn{4}{|c|}{ Late postoperative complications } \\
\hline redo TURP/VLP (n) & 5 & 0 & 0.02 \\
\hline incontinence (n) & 2 & 0 & 0.15 \\
\hline \multicolumn{4}{|c|}{ Complications based on Clavien-Dindo classification } \\
\hline minor complications (n) & 13 & 11 & 0.7 \\
\hline major complications (n) & 7 & 0 & 0.01 \\
\hline overall complications (n) & 20 & 11 & 0.06 \\
\hline
\end{tabular}

Table 3.

Functional results and PSA at 12 months.

\begin{tabular}{|lccc|}
\hline & TURP $(\mathbf{n}=\mathbf{5 0})$ & PVP $(\mathbf{n}=\mathbf{5 0})$ & $p$ value \\
\hline$Q_{\text {max }}(\mathrm{m} / \mathrm{s})$ & & & \\
preoperative & $7.6 \pm 3$ & $8.4 \pm 1.7$ & 0.14 \\
1 year & $15.6 \pm 6.4$ & $17 \pm 3$ & 0.15 \\
$\Delta \mathbf{Q}_{\text {max }}$ & $8.2 \pm 7.2$ & $9.7 \pm 2.1$ & 0.16 \\
\hline IPSS score & & & \\
preoperative & $20.1 \pm 5.4$ & $22.2 \pm 5.8$ & 0.06 \\
1 year & $8.7 \pm 5.1$ & $9.3 \pm 3$ & 0.58 \\
$\Delta$ IPSS & $10.7 \pm 6.8$ & $12.9 \pm 4.1$ & 0.052 \\
\hline PSA (ng/mL) & & & \\
preoperative & $2.29 \pm 2.19$ & $4.41 \pm 2.31$ & 0.001 \\
1 year & $1.9 \pm 2.8$ & $2.97 \pm 1.8$ & 0.03 \\
$\Delta$ PSA & $0.59 \pm 0.6$ & $1.73 \pm 1.4$ & 0.001 \\
& & & \\
\hline
\end{tabular}

cedures was similar and there were no statistically significant differences between the groups for the primary outcome functional parameters except for PSA degrees. The 1 year postoperative mean improvement in IPSS was $9.3 \pm 3$ in the PVP group and $8.7 \pm 5.1$ in the TURP group, and was not statistically different between the two groups ( $p=0.58$ ) and this reflected in the $Q_{\max }$ score. Reduction of PSA, that reflects the major reduction of prostate volume, was statistically major in VLP group respect TURP group $(\mathrm{p}=0.001)$ 


\section{Discussion}

For many years, TURP has been considered the gold standard for the treatment of LUTS secondary to benign prostatic hyperplasia. Very often, however, TURP is characterized by numerous post-operative complications. PVP was then introduced to minimize intra and post-operative complications with minimally invasive intent. The KTP 80-W laser is a light wavelength of 532 $\mathrm{nm}$, which is strongly absorbed by hemoglobin. The GreenLight XPS 180-W laser provides the same wavelength of $532 \mathrm{~nm}$ with similar intrinsic absorption characteristics (15).

As a result, laser prostate ablation has been developed to minimize the risk of bleeding complications. The reduction of complications then becomes particularly important in the older and fragile population that frequently carries coronary stents or who suffers from atrial fibrillation, coronary artery disease, valvular cardiopathies or deep vein thrombosis. These conditions are mainly managed through use of oral anticoagulants or antithrombotic agents (16). Laser light vaporizes the prostatic tissues layer by layer; the thickness of each layer is $1-2 \mathrm{~mm}$. At the same time, capillary vessels are coagulated to stop bleeding. This explains why the occurrence of intraoperative bleeding and blood transfusion is hardly seen in PVP treatment. In our study, the rate of transfusions and the reduction of hemoglobin is statistically lower in the PVP group as well and this is associated with a lower hospital stay and catheterization time (17). The catheterization and hospital time are important factors affecting patient's quality of life, so this result indicates a major advantage of PVP.

Considering complications the clot retention was the most frequent early complication in the TURP group (26\%) and this may occur as a consequence as well as premature termination of procedure, with consequent inadequate relief of obstruction: only 3 patients (6\%) experienced a recatheterization after PVP.

Large non randomised studies have reported a more favorable safety profile for green light than TURP regarding major complications (18) accordingly to our results (7 vs $0 ; \mathrm{p}<0,001$ ). In our study the reintervention rate was higher in the TURP group as respect to the PVP group and it was caused by bladder neck sclerosis or prostate tissue regrowth; another cause may be secondary to the reduced amount of tissue resected in patients undergoing TURP, which is underlined by the minor reduction of the postoperative PSA. One-year functional results showed dramatic improvement in both groups regarding reduction of IPSS and improvement of $\mathrm{Q}_{\max }$ with no significant difference between both groups. Only greater urgency in the PVP group should be reported in the immediate postoperative period.

This study reveals that PVP offers more advantages than TURP in terms of clot retention, blood transfusion and lower incidence rate of major complications.

Furthermore, compared to the TURP group, patients undergoing PVP have a shorter duration of catheterization, less blood loss and shorter hospital stays, which may explain the broad acceptance of PVP surgery from patients with secondary LUT to BPH, as confirmed by metanalysis (18-20). The benefits of laser-based prostat- ic surgery are reflected in its increasing use in daily clinical practice. The BPO rate treated with lasers increased from $6 \%$ in 2000 to $45 \%$ in 2011 (21). Since its introduction in 1995, for example, green lasers now account for about 23\% of BPH surgeries in France (22). Also, it is important to point out that there is a growing interest to improve the use of laser in order to preserve the antegrade ejaculation (23).

Our study has some limitations. First, this is a non-randomized study comparing different surgical approaches. However, many patients are unwilling to be randomly assigned to a particular surgical treatment and are usually attracted by the most modern surgical procedure or chose a procedure based on personal preferences for a specific surgeon. Second, our study involved a relatively low number of patients with inherent biases related to data collection. In this case, selection biases were partially limited by the inclusion criteria: the last consecutive 50 interventions for each surgical technique were included. Also, indications to surgery, operative techniques and perioperative management, and data collection should be relatively homogeneous in a single center. Furthermore, age, prostate volume, used of anticoagulants or antiplatelets, and ASA score were comparable between groups.

\section{Conclusions}

PVP has advantages in terms of perioperative safety and rate of major complications than TURP.

Functional outcomes at 1 year of follow-up were comparable.

\section{REFERENCES}

1. Berry SJ, Coffey DS, Walsh PC, Ewing LL The development of human benign prostatic hyperplasia with age. J Urol. 1984; 132:474-479.

2. Michel MC, Mehlburger L, Bressel HU, et al. Tamsulosin treatment of 19,365 patients with lower urinary tract symptoms: does comorbidity alter tolerability? J Urol. 1998; 160:784-791.

3. Kaplan SA. Re: EAUguidelines on the treatment and followup of non-neurogenic male lower urinary tract symptoms including benign prostatic obstruction. J Urol. 2014; 192:1167.

4. Holtgrewe HL, Mebust WK, Dowd JB, et al. Transurethral prostatectomy: practice aspects of the dominant operation in American urology. J Urol. 1989; 141:248-253.

5. Rassweiler J, Teber D, Kuntz R, Hofmann R. Complications of transurethral resection of the prostate (TURP)-incidence, management, and prevention. Eur Urol. 2006; 50:969-979.

6. Reich O, Gratzke C, Bachmann A, et al. Morbidity, mortality and early outcome of transurethral resection of the prostate: a prospective multicenter evaluation of 10,654 patients. J Urol. 2008; 180:246-249.

7. Borboroglu PG, Kane CJ, Ward JF, et al. Immediate and postoperative complications of transurethral prostatectomy in the 1990s. J Urol. 1999; 162:1307-1310.

8. Wasson JH, Reda DJ, Bruskewitz RC, et al. A comparison of transurethral surgery with watchful waiting for moderate symptoms of benign prostatic hyperplasia. The Veterans Affairs Cooperative 
Study Group on Transurethral Resection of the Prostate. N Engl J Med. 1995; 332:75-79.

9. Watson G. Contact laser prostatectomy. World J Urol. 1995; 13:115-118

10. Rieken M, Bonkat $G$, Müller $G$, et al. The effect of increased maximum power output on perioperative and early postoperative outcome in photoselective vaporization of the prostate. Lasers Surg Med. 2013; 45:28-33.

11. Bouchier-Hayes DM, Van Appledorn S, Bugeja P, et al. A randomized trial of photoselective vaporization of the prostate using the 80-W potassium-titanylphosphate laser vs transurethral prostatectomy, with a 1-year followup. BJU Int. 2010; 105:964-969.

12. Thomas JA, Tubaro A, Barber N, et al. A multicenter randomized non-inferiority trial comparing GreenLight-XPS laser vaporization of the prostate and transurethral resection of the prostate for the treatment of benign prostatic obstruction: two-yr outcomes of the goliath study. Eur Urol. 2016; 69:94-102.

13. Reich O, Bachmann A, Siebels M, et al. High power ( 80 W) potassium-titanyl-phosphate laser vaporization of the prostate in 66 high risk patients. J Urol. 2005; 173:158-160.

14. Dindo D, Demartines N, Clavien P.A. Classification of Surgical Complications: a new proposal with evaluation in a cohort of 6336 Patients and results of a survey. Ann Surg. 2004; 240:2.

15. Malek RS, Kang HW, Peng YS, et al. Photoselective vaporization prostatectomy: experience with a novel $180 \mathrm{~W} 532 \mathrm{~nm}$ lithium triborate laser and fiber delivery system in living dogs. J Urol. 2011; 185:712-8

16. Whitlock EP, Burda BU, Williams SB, et al. Bleeding risks with aspirin use for primary prevention in adults: a systematic review for the U.S. preventive services task force. Ann Intern Med. 2016; 164:826.

17. Van Cleynenbreugel B, Srirangam SJ, Van Poppel $H$. Highperformance system GreenLight laser: indications and outcomes. Curr Opin Urol. 2009; 19:33-37.

18. Teng J, Zhang D, Li Y, et al. Photoselective vaporization with the green light laser vs transurethral resection of the prostate for treating benign prostate hyperplasia: a systematic review and metaanalysis. BJU Int. 2013; 111:312-323.

19. Ding H, Du W, Lu ZP, et al. Photoselective green-light laser vaporization vs. TURP for BPH: meta-analysis. Asian J Androl. 2012; 14:720-5.

20. Thangasamy IA, Chalasani V, Bachmann A, Woo HH. Photoselective vaporization of the prostate using $80-W$ and $120-W$ laser versus transurethral resection of the prostate for benign prostatic hyperplasia: a systematic review with metaanalysis from 2002 to 2012. Eur Urol. 2012; 62:315-23.

21. Chughtai BI, Simma-Chiang V, Lee R, et al. Trends and utilization of laser prostatectomy in ambulatory surgical procedures for the treatment of benign prostatic hyperplasia in New York state (2000- 2011). J Endourol. 2015; 29:700-706.

22. Peyronnet B, Cornu J-N, Rouprêt M, et al. Trends in the use of the GreenLight laser in the surgical management of benign prostatic obstruction in France over the past 10 years. Eur Urol. 2015; 67:1193-1195.

23. Leonardi $R$. The LEST technique: Treatment of prostatic obstruction preserving antegrade ejaculation in patients with benign prostatic hyperplasia. Arch Ital Urol Androl. 2019; 91:35-42.

\section{Correspondence}

Daniele Mattevi, MD, FEBU (Corresponding author)

danielemattevi85@gmail.com

Lorenzo Giuseppe Luciani, MD

lorenzo_luciani@hotmail.com

Rosa Spina, MD

rosa.spina@apss.tn.it

Claudio Divan, MD

claudio.divan@apss.tn.it

Stefania Cicuto, MD

stefania.cicuto@apss.tn.it

Tommaso Cai, MD

tommaso.cai@apss.tn.it

Valentino Vattovani, MD

valentino.vattovani@apss.tn.it

Marco Puglisi, MD

marco.puglisi@apss.tn.it

Chiodini Stefano, MD

Gianni Malossini, MD

gianni.malossini@apss.tn.it

Department of Urology, Santa Chiara Hospital

Largo Medaglie d'oro 9, 38122, Trento, Italy 